\title{
Randomized Efficacy and Safety Trial with Oral S 44819 after Recent ischemic cerebral Event (RESTORE BRAIN study): a placebo controlled phase II study
}

Hugues Chabriat ${ }^{1,2,3}$, Claudio L. Bassetti ${ }^{4}$, Ute Marx ${ }^{5}$, Françoise Picarel-Blanchot ${ }^{5}$, Aurore Sors ${ }^{5}$, Celine Gruget ${ }^{5^{*}}$, Barbara Saba ${ }^{5}$, Marine Wattez ${ }^{5}$, Marie-Laure Audoli5 ${ }^{5}$ and Dirk M. Hermann ${ }^{6}$

\begin{abstract}
Background: The GABA $A_{A}-a 5$ receptor antagonist $\$ 44819$ is a promising candidate to enhance functional recovery after acute ischemic stroke (IS). S44819 is currently evaluated in this indication; RESTORE brain study started in Dec 2016 and was completed in March 2019.

Methods/design: The study is a 3-month international, randomized, double-blind, parallel group, placebocontrolled phase II multicentre study. Patients in 14 countries who suffered an IS leading to a moderate or severe deficit defined by NIHSS score ranging from 7 to 20 and are aged between 18 to 85 years are included between 3 and 8 days after the stroke onset. Approximately 580 patients are to be included.

The primary objective of the study is to demonstrate the superiority of at least one of the two doses of S44819 (150 or $300 \mathrm{mg}$ bid) compared to placebo on top of usual care on functional recovery measured with the modified Rankin scale at 3 months. Comparisons between two doses of S44819 and placebo are assessed with ordinal logistic regression evaluating the odds of shifting from one category to the next in the direction of a better outcome at day 90. Secondary objectives include the evaluation of S44819 effects on neurological examination using the National Institute of Health Stroke Scale total score, activities of daily living using the Barthel Index total score, and cognitive performance using the Montreal Cognitive Assessment scale total score and Trail Making Test times. Safety and tolerability of the two doses of S44819 will also be analyzed.

Discussion: The RESTORE BRAIN study might represent the first proof of concept study of an innovative therapeutic approach that is primarily based on enhancing functional recovery after IS.

Trial registration: Randomized Efficacy and Safety Trial with Oral S 44819 after Recent ischemic cerebral Event, an international, multi-centre, randomized, double-blind placebo-controlled phase II study. ClinicalTrials.gov, NCT02 877615; Eudract 2016-001005-16. Registered 24 August 2016
\end{abstract}

Keywords: S44819, Ischemic cerebral stroke, Efficacy, Safety, Study design, GABA

\footnotetext{
* Correspondence: celine.gruget@servier.com

${ }^{5}$ Institut de Recherches Internationales Servier (IRIS), 50 rue Carnot, 92284

Suresnes Cedex, France

Full list of author information is available at the end of the article
}

(c) The Author(s). 2020 Open Access This article is distributed under the terms of the Creative Commons Attribution 4.0 International License (http://creativecommons.org/licenses/by/4.0/), which permits unrestricted use, distribution, and reproduction in any medium, provided you give appropriate credit to the original author(s) and the source, provide a link to the Creative Commons license, and indicate if changes were made. The Creative Commons Public Domain Dedication waiver (http://creativecommons.org/publicdomain/zero/1.0/) applies to the data made available in this article, unless otherwise stated. 


\section{Background}

Post-stroke recovery relies on brain neuroplasticity [1, 2], which is compromised by the sustained hypoexcitability observed in the peri-infarct cortex due to the increased activity of GABAergic neurons [3].

S44819 is a potent and selective antagonist of $\mathrm{GABA}_{\mathrm{A}^{-}}$ $\alpha 5$ receptors [4] that enhances motor and cognitive recovery when administered chronically from day 3 after IS in rodent models [5]. A transcranial magnetic stimulation study has demonstrated in humans that S44819 reaches the human cortex and is capable of increasing cortical and cortico-spinal excitability by reducing $\mathrm{GABA}_{\mathrm{A}}$ receptor-mediated activity [6].

The design of a phase II clinical trial testing the efficacy of two doses of S44819 on functional recovery after acute supratentorial IS is presented.

\section{Methods/design}

\section{Design}

In accordance with the EMA's guidance on stroke [7], the study includes a selection period and a double-blind treatment period of 90 days. A follow-up period of 15 days after the end of treatment allows evaluation of the safety of patients (Fig. 1).

\section{Patient population}

Male or female patients aged $18-85$ years are randomised between 3 and 8 days after the stroke event based on the following criteria: 1) acute IS confirmed by MRI or CT; 2) NIHSS score between 7 and 20 both inclusive $[8,9]$; 3) lack of previous disability defined as an estimated prestroke mRS score $0-1$; 4) written informed consent.

Patients are ineligible if: 1) an acute hemorrhagic stroke or symptomatic hemorrhagic transformation or cerebral venous thrombosis occurs; 2) the required rehabilitation is impossible to undertake; 3 ) a carotid endarterectomy is planned within the next 3 months; 4) a previous clinically significant condition interferes with the study evaluation; 5) brain imaging shows a severe microangiopathy (Fazekas grade 3 with severe small vessels disease on MRI or CT scan); 6) receiving a treatment that could have an impact on treatment efficacy (i.e., interacting with GABA-A receptors) or a clinically relevant abnormality likely to interfere with study outcome, such as any renal or hepatic severe impairment and any repeated QTcF prolongation. Patients who are not able to cooperate as well as those presenting with geographic or social factors making their study participation impossible are excluded.

\section{Setting}

The study is conducted in 92 hospital stroke centers in 14 countries (Australia, Belgium, Brazil, Canada, Czech Republic, France, Germany, Hungary, Italy, Poland, South Korea, Spain, The Netherlands, United Kingdom) that have experienced specialists in stroke medicine (e.g., are able to perform intravenous thrombolytic therapy, brain imaging for diagnosis). At discharge from hospital stroke centers, patients receive rehabilitation therapy (in- or out-patient rehabilitation) in accordance with the standard of care

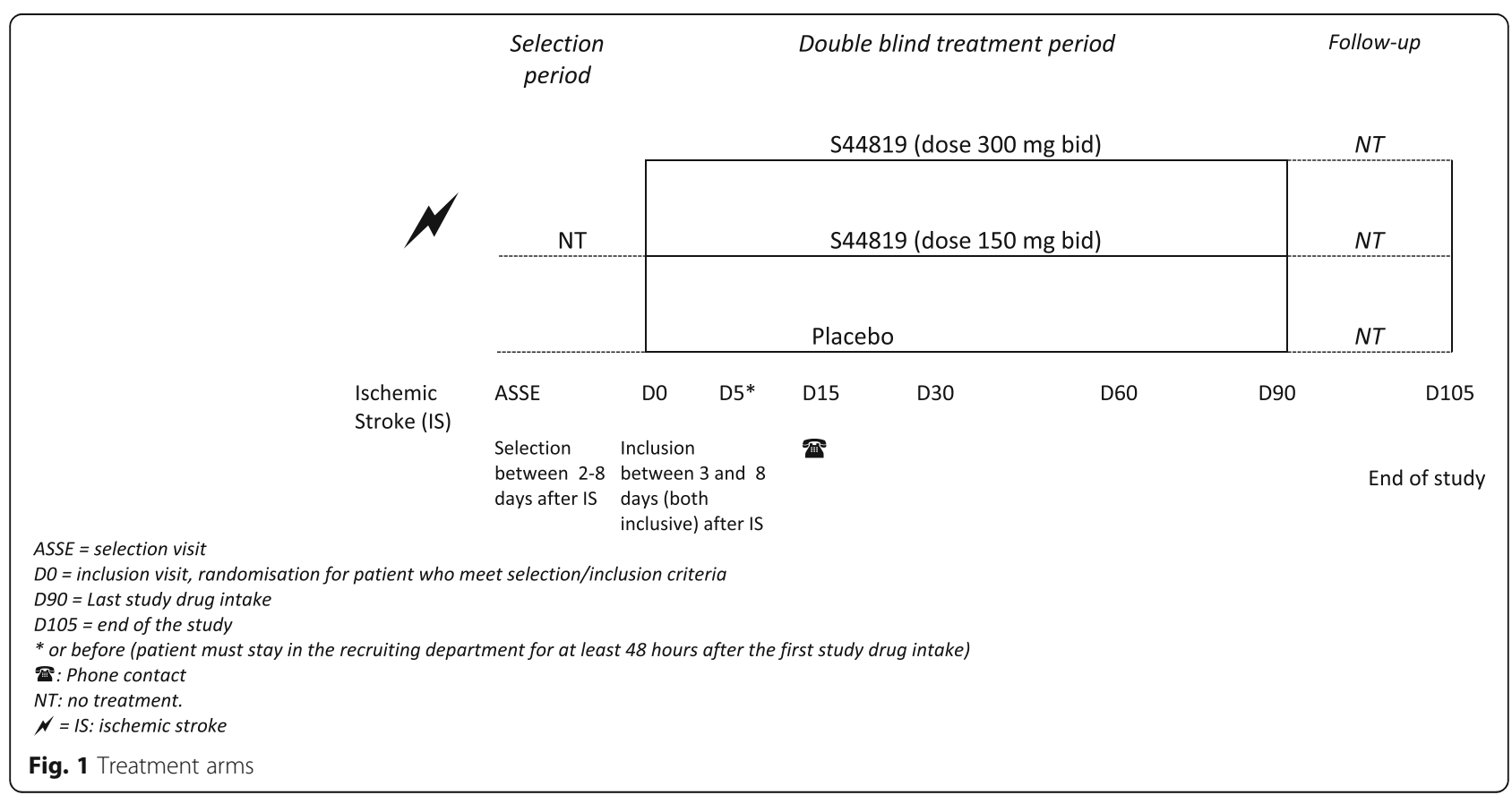


in each country and their clinical status. Patients are followed by the enrolling investigators.

\section{Randomization}

The treatment is assigned at the inclusion visit by a balanced, non-adaptive randomization, with stratification by country and previous revascularization therapy status (thrombolysis and/or endovascular therapy). Patients are randomized into one of the three groups: S 44819 (150 $\mathrm{mg}$ or $300 \mathrm{mg}$ twice a day) or placebo to reach about 194 patients per treatment arm. Randomization and allocations are centralized by Interactive Web Response System (IWRS) under blind conditions for subjects, caregivers, investigators, study-related staff, and sponsor. The placebo is made up of hydroxypropyl methylcellulose acetate succinate and different iron oxide colorants and is an off-white to yellow powder in a sealed sachet for oral suspension. Investigational Medicinal Product (IMP) is provided in the form of sachets (two sachets per intake) with an identical appearance and taste for all treatment groups. The circumstances under which blinding may be broken in IWRS are any serious AEs or severe medical conditions where the knowledge of the treatment is necessary for safety follow-up of the patient.

The Methodology and Analysis of Clinical Data Division of I.R.I.S is responsible for designing and constructing the blinded randomization list.

\section{Treatment}

Study treatments are S 44819 (150 or $300 \mathrm{mg} / \mathrm{bid})$ and placebo. The choice of doses is mainly based on a Transcranial Magnetic Stimulation study which has demonstrated that S 44819-at least at doses $>100$ $\mathrm{mg}$-reaches the human cortex and increases corticospinal excitability by reducing $\mathrm{GABA}_{\mathrm{A}} \alpha 5$-mediated inhibition [6].

As no validated post-acute phase treatment exists to improve functional recovery after stroke events, no active comparator is available. In this context, a placebo comparator group is commonly employed and required by the guidelines to demonstrate efficacy in controlled clinical trials [10].

During the double-blind treatment period (D0 to D90), S 44819 or placebo is provided in the form of sachets (two in the morning and two in the evening) of identical appearance and taste for all treatment groups. Patients remain on the same IMP and dose throughout the treatment period. Depending on the patient's condition, different methods of treatment administration are planned, e.g., with a glass of water or thickened water, if necessary through a nasogastric tube.

Criteria for discontinuing treatment are any adverse event according to the judgment of the investigator, any
QTcF prolongation, any severe hepatic event, any suicide attempt, any symptomatic haemorrhagic stroke, or any new IS. Also, consent withdrawal or any event which could jeopardize the patient's safety lead to treatment discontinuation. In such a situation, the withdrawal reason is reported and all examinations are expected to be performed.

Compliance with treatment is assessed at each study visit by deduction of the number of sachets dispensed and returned.

\section{Endpoints and measurements}

The primary objective is to demonstrate the superiority of at least one of the two doses of S 44819 versus placebo on functional recovery after IS based on the modified Rankin Scale (mRS) [11] measured after 90 days of treatment. The $\mathrm{mRS}$ is administered at days 5, 30, 60, and 90 and at the follow-up visit (day 105).

The secondary objectives are to assess the efficacy of two doses of S 44819 versus placebo on stroke recovery using the National Institute of Health Stroke Scale (NIHSS) [12], on activities of daily living using Barthel Index (BI) total scores [13], and on cognitive performance tests (MoCA, TMT), as well as the safety and tolerability of S 44819.

The NIHSS is administered at selection visit, at baseline (day 0, inclusion), and at days 5, 30, 60, 90, and 105 . The BI is administered at days 30, 60, 90, and 105 .

Cognitive performance is assessed using the Montreal Cognitive Assessment scale (MoCA) [14] and Trail Making Test (TMT) [15]. The MoCA (total score) and TMT (A and $\mathrm{B}$, times) results are obtained at days 30 and 90 . In order to evaluate cognitive performances when the condition begins to stabilize, cognitive assessment is obtained at 1 month and 3 months to assess the course of cognitive impairment in each stroke patient.

A visual analog scale evaluates various sub-dimensions of the participant's quality of life (appetite, sleep, daytime alertness, mood, anxiety, and pain).

Safety and tolerability assessment includes adverse event (AE) recording, vital signs, and physical examination at all visits; body weight at selection, days 30, 60, 90, and 105, BMI and ECG parameters at days 0, 5, 30, 60,90 , and 105 , and different laboratory parameters at days $0,30,60,90$, and 105 .

Suicidal ideation and suicidal behavior is assessed using the Columbia Suicide Severity Rating Scale (CSSRS) [16] and is administered at each visit from days 5 to 105 .

Measurements are also performed in case of premature withdrawal.

A SPIRIT figure is shown in Fig. 2 and a SPIRIT checklist is available in Additional file 1. 


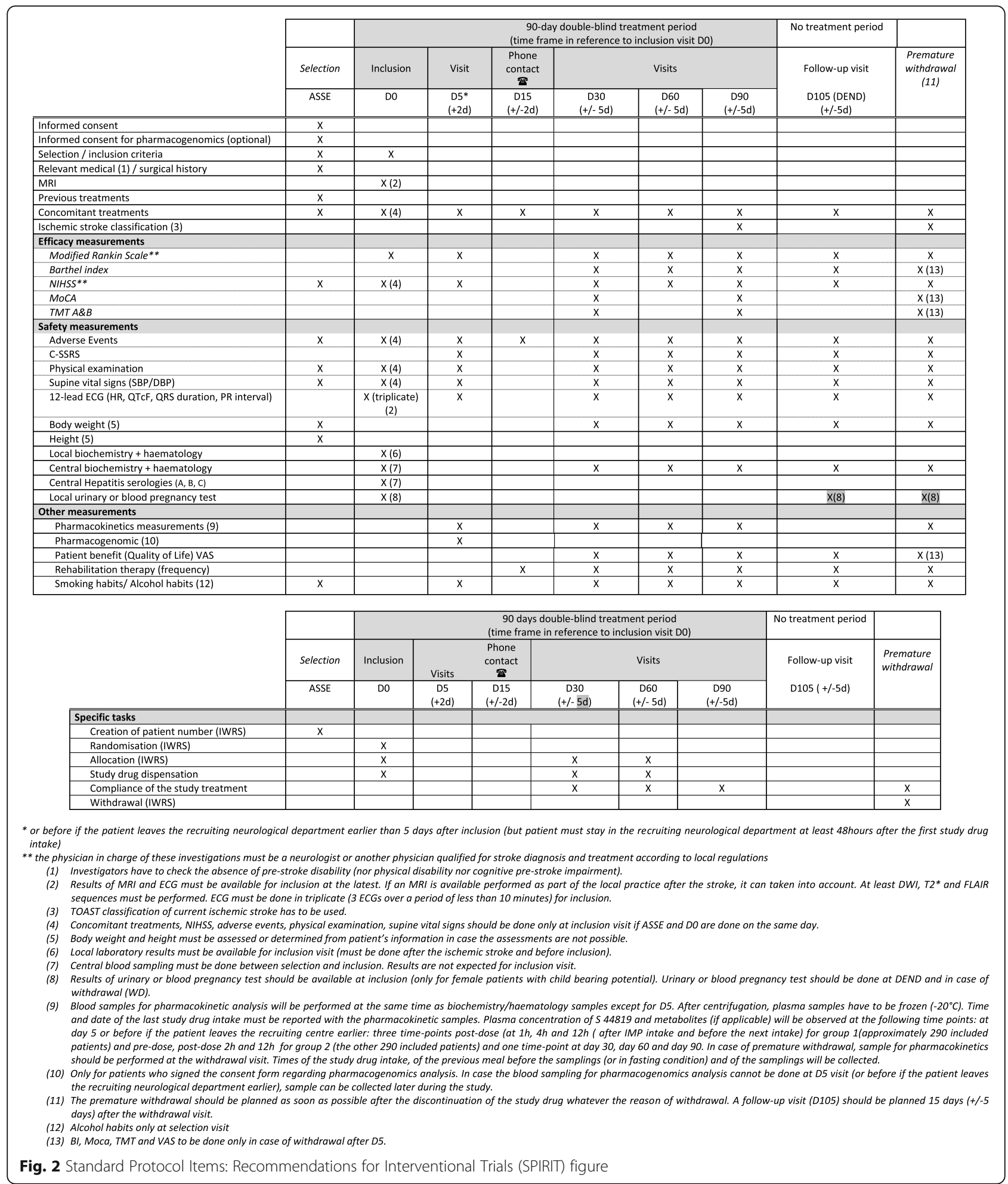

\section{Sample size estimates}

The sample size (580 patients) was estimated based on the mRS score at day 90, using the last observation carried forward (LOCF) approach, to detect a treatment effect between at least one dose of S 44819 and placebo in the full analysis set (FAS) using Whitehead's formula [17] for ordered categorical criteria. To maintain the experiment-wise type I error at 5\%, the Bonferroni correction is to be applied. A drop-out rate of 5\% until D5 was considered. This sample size 
should allow reaching the primary objective with a power of $85 \%$ if the true difference is 15 points in the cumulative proportion of patients with $\mathrm{mRS} 0-2$ after 90 days of treatment and considering the following distribution of $\mathrm{mRS}$ assumed for placebo after 90 days of treatment:

\begin{tabular}{lllllll}
\hline mRS & 0 & 1 & 2 & 3 & 4 & $5-6$ \\
\hline Placebo (\%) & 10 & 15 & 20 & 20 & 20 & 15 \\
\hline
\end{tabular}

The placebo distribution was adapted from the NEST1 trial [18] with similar inclusion criteria concerning severity (NIHSS score between 7 and 22). The results of NEST-1 show that such a placebo distribution is a reasonable estimation.

\section{Statistical analysis \\ Main analysis}

The primary efficacy objective is assessed in the FAS (all patients of the RS=Randomised Set having taken at least one dose of treatment and having at least a value for the primary efficacy endpoint after D5) from the mRS score at day 90. An ordinal logistic regression assesses the odds of shifting from one category to the next on the mRS scale. Analysis includes the fixed, categorical effects of treatment (including the three treatment groups), country, and previous revascularisation therapy. Missing data are imputed with the LOCF approach. The stepdown Dunnett procedure accounts for multiplicity of comparisons.

To assess the robustness of the primary analysis results with regard to the method of handling missing data, an ordinal logistic regression, including the fixed, categorical effects of treatment, country, and previous revascularisation therapy, is carried out in the FAS considering the multiple imputation method. The same analysis as the primary analysis is performed in the FAS, including in addition the continuous fixed, covariates of age, and baseline NIHSS score. Multiplicity is handled in the same way as in the primary analysis.

\section{Secondary analyses}

The same analyses as for the primary analysis are performed in patients of the per protocol set (all completed patient data of the FAS without relevant deviation). The difference between each S 44819 dose and placebo is studied in the FAS at day 90 on the dichotomized mRS scores $(0-1$ versus $2-6$ and $0-2$ versus 3-6) using a logistic regression including the fixed, categorical effect(s) of treatment, country, and previous revascularisation therapy. Missing data at day 90 are imputed with the LOCF approach. The step- down Dunnett procedure is used to account for multiplicity of comparisons.

Descriptive statistics by treatment group are provided for all analytical approaches of the primary efficacy endpoint in patients of the FAS. The mRS score is described at each visit in patients of the per protocol set.

Secondary endpoints The secondary efficacy endpoints include 1) the value of the NIHSS score at baseline and at each post-baseline visit, 2) the value of the BI total score at each visit, 3) the value of the MoCA total score at each visit, and 4) the TMT times at each visit.

The difference between each S 44819 dose and placebo is studied in the FAS at day 90 for the NIHSS and BI scores using a Mann-Whitney test. Missing data at day 90 are imputed with the LOCF approach. Descriptive statistics by treatment group are provided for the NIHSS, BI, MoCA, and TMT endpoints in patients of the FAS.

Number of events and the number and percentage of patients reporting at least one adverse event are provided for serious and emergent adverse events. These events are described according to the seriousness, intensity, relationship, action taken regarding $\mathrm{S} 44819$, the requirement of added therapy, time from onset, and outcome.

Vital signs, laboratory parameters, ECG parameters, and physical examination are described in terms of value at baseline, value at each post-baseline visit under treatment, last post-baseline values under treatment, and values at follow-up visit, as well as variations from baseline to each post-baseline visit under treatment and to the last post-baseline value under treatment.

For suicidal ideation score from the C-SSRS, the number and percentage of patients is assessed considering their maximum suicidal ideation score during treatment. Suicidal ideation score is also described at follow-up visit. For the other outcomes, the number and percentage of patients that reach each outcome during treatment (defined as a "yes" answer at any time during treatment) and at follow-up visit are described.

Patient quality of Life (QoL) is scored at each visit and descriptive statistics are reported for visual analogue scale scores for the D0-D90 period as well as at day 105.

Statistical analysis is performed using $\mathrm{SAS}^{\odot}$ software, version 9.2 (Cary, NC, USA).

\section{Data Monitoring Committee}

1A Data Monitoring Committee (DMC) is responsible for reviewing on a regular basis strictly confidential data related to the safety of patients participating in the study. Based on the review of the safety data, the DMC gives written recommendations to the sponsor about the continuation of the study per the protocol 
until the next DMC meeting, or about the continuation of the study with modification(s) that have no impact on the study design, or about the premature discontinuation of the study.

\section{Discussion}

S 44819 exerts long lasting improvement of post-stroke recovery in rodent IS models when administered from day 3 after the ischemic insult [5] and has been shown to increase cortical excitability by decreasing GABAmediated inhibition in healthy volunteers [6]. It is hypothesized that $S 44819$ may enhance post-stroke neuroplasticity in patients by counteracting enhanced cortical tonic inhibition.

Accumulating evidence suggests that there is a critical period of increased neuroplasticity during the early poststroke recovery phase $[3,19]$ and it is crucial to initiate therapy during this time window. In this trial, S 44819 is administered to patients starting 3 to 8 days after stroke onset.

The study protocol considers all ethical principles of a placebo-controlled trial and the best standards of care. To increase the acceptability of the present study, the probability to receive placebo is fixed at $33 \%$. Given that S 44819 is expected to improve recovery in IS patients regardless of their initial condition, a shift analysis is recommended [20]. Comparisons between S 44819 and placebo are assessed using ordinal logistic regression to assess the odds of shifting from one category to the next.

The mRS, NIHSS, and BI scales are analyzed as secondary efficacy scales; they are recommended by EMA guidelines to assess the efficacy of medicinal products for treating acute stroke [7].

S 44819 does not bind to benzodiazepine sites or to $\mathrm{GABA}_{\mathrm{A}}$ receptors containing $\alpha 1, \alpha 2$, and $\alpha 3$ subunits [4] and presumably does not cause adverse effects triggered by these subunits; so far, no safety concern has arisen from the phase I and the on-going phase II study.

\section{Conclusion}

This trial may provide the first proof of concept for an innovative therapeutic approach based on the enhancement of functional recovery after IS.

\section{Trial status}

Protocol version 7, 23/07/2018. The study started in Dec 2016 and was completed in March 2019.

\section{Supplementary information}

Supplementary information accompanies this paper at https://doi.org/10. 1186/s13063-020-4072-2.

Additional file 1. SPIRIT 2013 checklist: Recommended items to address in a clinical trial protocol and related documents.

\section{Abbreviations}

AE: Adverse events; BI: Barthel Index; C-SSRS: Columbia Suicide Severity Rating Scale; FAS: Full analysis set; IS: Ischemic stroke; IWRS: Interactive Web Response System; LOCF: Last observation carried forward; MoCA: Cognitive Assessment scale; mRS: Modified Rankin Scale; NIHSS: National Institute of Health Stroke Scale; QoL: Quality of Life; QTCF: QT interval corrected using Fridericia formula for heart rate; TMS: Transcranial magnetic simulation; TMT: Trail Making Test

\section{Acknowledgements}

Not applicable.

\section{Authors' contributions}

$\mathrm{UM}, \mathrm{FPB}, \mathrm{CGP}$, and BS designed the study, wrote the protocol, and managed the literature searches. All authors contributed to the first draft of the manuscript and have approved the final manuscript. All authors read and approved the final manuscript.

\section{Funding}

This study was sponsored by Servier (Suresnes, France).

\section{Availability of data and materials}

Anonymized patient-level, study-level clinical trial data (including clinical study report) and study protocol underlying the results reported in this article will be shared in agreement with the Servier Data Sharing Policy available at https://clinicaltrials.servier.com/data-request-portal/.

Access to data will be granted to researchers identified in the research proposal directed to https://clinicaltrials.servier.com/data-request-portal/, to achieve the aims described in this proposal, and provided it is approved by a dedicated committee and a signed data sharing agreement by the requestor is provided.

\section{Ethics approval and consent to participate}

The study is performed in accordance with the ethics principles stated in the Declaration of Helsinki 1964, as revised in Fortaleza, 2013.

The study protocol, the "Participant information and consent form" document, the "Authorised representative according to local regulations information and consent form" document, the list of investigators document, the insurance documents, the investigator's brochure of S 44819 are be submitted to (an) Institutional Review Board(s)/Independent Ethics Committee(s) by the investigator(s) or the national coordinator(s) or the sponsor in accordance with local regulations. The study does not start in a centre before written approval by corresponding Institutional Review Board(s)/Independent Ethics Committee(s) has been obtained, the local regulatory requirements have been complied with, and the signed clinical study protocol of each contractual party involved has been obtained. The investigator or a person designated by him/her is to collect written consent from each participant before his/her participation in the study.

\section{Competing interests}

C. Bassetti, H. Chabriat, and D. Hermann declare no competing interests. U. Marx, F. Picarel-Blanchot, A. Sors, C. Gruget-Petazzoni, B. Saba, M. Wattez, and M.L. Audoli are employees at Servier.

\section{Author details}

'Department Neurology, Lariboisière Hospital, APHP and University Denis Didierot, Paris 7, Paris, France. ${ }^{2}$ FHU Neuro Vasc, Paris, France. ${ }^{3}$ INSERM U1141, Paris, France. ${ }^{4}$ Inselspital Bern, Bern, Switzerland. ${ }^{5}$ Institut de Recherches Internationales Servier (IRIS), 50 rue Carnot, 92284 Suresnes Cedex, France. ${ }^{6}$ Chair of Vascular Neurology, Dementia and Ageing Research, Department of Neurology, University Hospital Essen, Hufelandstraße 55, 45122 Essen, Germany.

Received: 7 November 2018 Accepted: 13 January 2020

Published online: 03 February 2020

References

1. Hermann DM, Chopp M. Promoting brain remodelling and plasticity for stroke recovery: therapeutic promise and potential pitfalls of clinical translation. Lancet Neurol. 2012;11(4):369-80. 
2. Murphy TH, Corbett D. Plasticity during stroke recovery: from synapse to behaviour. Nat Rev Neurosci. 2009;10(12):861-72.

3. Carmichael ST. Brain excitability in stroke: the yin and yang of stroke progression. Arch Neurol. 2012;69(2):161-7.

4. Etherington $L A$, Mihalik B, Palvolgyi A, et al. Selective inhibition of extrasynaptic alpha5-GABAA receptors by $S$ 44819, a new therapeutic agent. Neuropharmacology. 2017;125:353-64.

5. Wang Y-C, Sanchez-Mendoza E, Silva de Carvahlo T, et al. Post-acute delivery of GABAA receptor alpha5-antagonist $S 44819$ promotes motorcoordination recovery, prevents secondary brain atrophy and reduces astrocytic scar formation after transient middle cerebral artery occlusion in mice [abstract]. In: Annual meeting of the American Academy of Neurology; 2017.

6. Darmani G, Zipser CM, Bohmer GM, et al. Effects of the selective alpha5GABAAR antagonist $S 44819$ on excitability in the human brain: a TMS-EMG and TMS-EEG phase I study. J Neurosci. 2016;36(49):12312-20.

7. EMA Guideline. Points to consider on clinical investigation of medicinal products for the treatment of acute stroke. CPMP/EWP/560/98; 2001. p. 1-7.

8. Adams HP, Davis PH, Leira EC, Chang K-C, Bendixen BH, Clarke WR, Woolson RF, Hansen MD. Baseline NIH Stroke Scale score strongly predicts outcome after stroke: a report of the Trial of Org 10172 in Acute Stroke Treatment (TOAST). Neurology. 1999;53:126-31.

9. Wouters A, Nysten C, Thijs V, Lemmens R. Prediction of outcome in patients with acute ischemic stroke based on initial severity and improvement in the first 24 h. Front Neurol. 2018;9:308.

10. ICH E10 : choice of control group and related issues in clinical trials.CPMP/ ICH/364/96; 2001. p. 1-30.

11. Rankin J. Cerebral vascular accidents in patients over the age of 60. II. Prognosis. Scott Med J. 1957;2(5):200-15.

12. Schlegel DJ, Tanne D, Demchuk AM, Levine SR, Kasner SE. Prediction of hospital disposition after thrombolysis for acute ischemic stroke using the National Institutes of Health Stroke Scale. Arch Neurol. 2004;61 (7):1061-4.

13. Mahoney F, Barthel D. Functional evaulation: the barthel index. Md State Med J. 1965;14:61-5.

14. Nasreddine ZS, Phillips NA, Bedirian V, et al. The Montreal Cognitive Assessment, MoCA: a brief screening tool for mild cognitive impairment. J Am Geriatr Soc. 2005;53(4):695-9.

15. Reitan RM. The relation of the trail making test to organic brain damage. $J$ Consult Psychol. 1955;19(5):393-4.

16. Posner K, Brown GK, Stanley B, et al. The Columbia-Suicide Severity Rating Scale: initial validity and internal consistency findings from three multisite studies with adolescents and adults. Am J Psychiatry. 2011;168(12):1266-77.

17. Whitehead J. Sample size calculations for ordered categorical data. Stat Med. 1993;12(24):2257-71.

18. Lampl Y, Zivin JA, Fisher M, Lew R, Welin L, Dahlof B, Borenstein P, Andersson B, Perez J, Caparo C, llic S, Oron U. Infrared laser therapy for ischemic stroke: a new treatment strategy: results of the NeuroThera Effectiveness and Safety Trial-1 (NEST-1). Stroke. 2007;38:1843-9.

19. Biernaskie J, Chernenko G, Corbett D. Efficacy of rehabilitative experience declines with time after focal ischemic brain injury. J Neurosci. 2004;24(5): 1245-54.

20. Saver JL, Gornbein J. Treatment effects for which shift or binary analyses are advantageous in acute stroke trials. Neurology. 2009;72(15):1310-5.

\section{Publisher's Note}

Springer Nature remains neutral with regard to jurisdictional claims in published maps and institutional affiliations.

Ready to submit your research? Choose BMC and benefit from:
- fast, convenient online submission
- thorough peer review by experienced researchers in your field
- rapid publication on acceptance
- support for research data, including large and complex data types
- gold Open Access which fosters wider collaboration and increased citations
- maximum visibility for your research: over 100M website views per year
At BMC, research is always in progress.
Learn more biomedcentral.com/submissions

